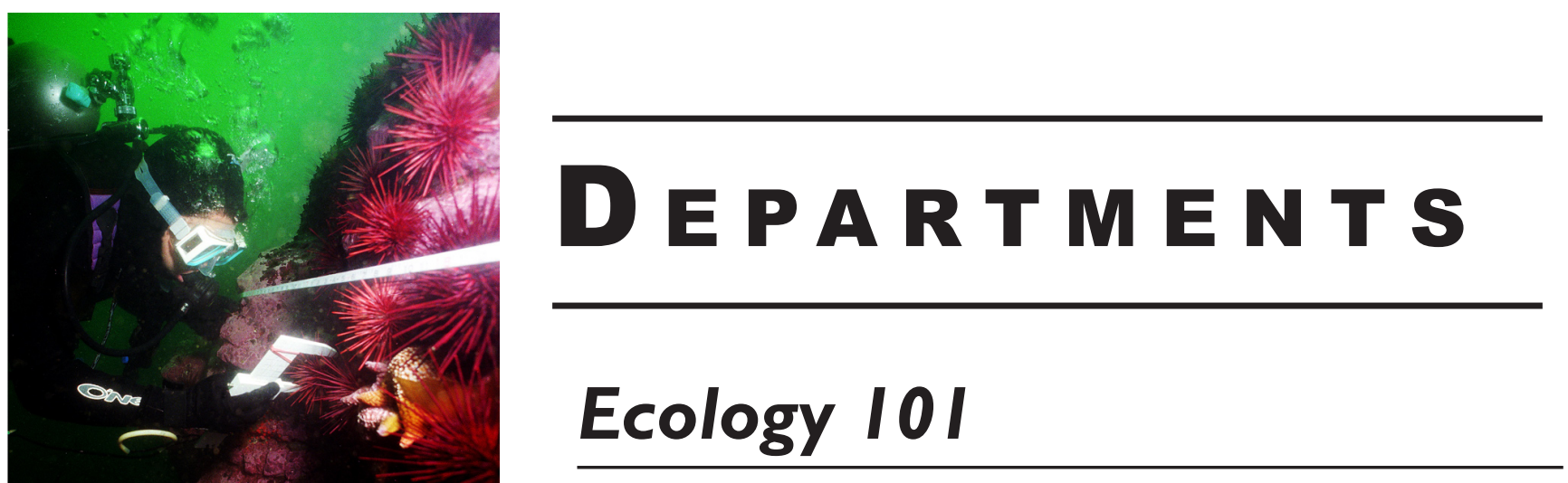

\title{
Statistics Without Math
}

Magnusson, W. E., and G. Mourão. 2004.

Statistics without math.

Sinauer Associates, Londrina, Brasil.

"Statistics without Math" sounds like an oxymoron, but it is actually an innovative text that empowers ecology students with a simple, clear conceptual understanding of deductive/inferential statistics. In the new millennium, students rarely encounter maths, as it is generally contained within the computer programs that perform their statistical analyses. Unlike most statistics books that leave the reader confused and bamboozled with mathematical jargon, this book focuses on providing the conceptual basis for inferential statistical analyses, ranging from the basic chisquare and $t$ test and ANOVA through to multivariate statistics and path analysis. These explanations allow students to understand how the computer has processed their data (without the algorithms).

There is little dispute that most undergraduate students in biology fear and loathe statistics. Overcoming "stats phobia" is a constant challenge for teachers and students alike. This book also forms the basis for an innovative course in Experimental Design and Statistics. It has been taught extensively, as a 2-week intensive course, at universities in Brazil, North America, Australia, and Fiji. The course is equally valuable for all students in the early stages of their research careers (honors, M.Sc. and Ph.D students), but is also valuable for managers and state agencies, providing them with a basis for understanding the statistics used in scientific reporting. Tips for teachers (Chapter 14) are available online at: 〈www.sinauer.com/swm

The book is relatively small (130 pages) making it an easy read for students, who readily consume it from cover to cover. While brief, it provides students with an understanding of statistics that leaves them with a thirst for more knowledge - and the confidence to face their demons, and tackle the stats books with the mathematics.

In summary, this book provides a novel format for learning statistics. It also provides the foundations for an elementary course for teaching statistics. As such it is an excellent tool, and an essential asset, for all students, teachers, and practitioners in biological sciences.

Jean-Marc Hero Endangered Frog Research Centre Centre for Innovative Conservation Strategies School of Environmental and Applied Sciences Griffith University, PMB 50

Gold Coast Mail Centre Queensland 9726, Australia 